\title{
GLAD!
}

Revue sur le langage, le genre, les sexualités

\section{Genre et discours métaphoriques sur la traduction}

Gender and the Metaphorics of Translation

\section{Lori Chamberlain}

Traducteur : Samantha Saïdi

\section{(2) OpenEdition}

\section{Journals}

Édition électronique

URL : http://journals.openedition.org/glad/2057

DOI : $10.4000 /$ glad.2057

ISSN : 2551-0819

Éditeur

Association GSL

Référence électronique

Lori Chamberlain, «Genre et discours métaphoriques sur la traduction », GLAD! [En ligne], 09 | 2020, mis en ligne le 20 décembre 2020, consulté le 20 janvier 2021. URL : http://journals.openedition.org/ glad/2057 ; DOl : https://doi.org/10.4000/glad.2057

Ce document a été généré automatiquement le 20 janvier 2021.

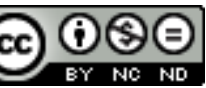

La revue GLAD! est mise à disposition selon les termes de la Licence Creative Commons Attribution Pas d'Utilisation Commerciale - Pas de Modification 4.0 International. 


\title{
Genre et discours métaphoriques sur la traduction
}

\author{
Gender and the Metaphorics of Translation
}

\author{
Lori Chamberlain
}

Traduction : Samantha Saïdi

\section{NOTE DE L'AUTEUR}

Toute ma reconnaissance et mes remerciements vont aux nombreuses amies et conversations qui ont aidé à clarifier ma pensée sur le sujet de cet article : Nancy Armstrong, Michael Davidson, Page duBois, Julie Hemker, Stephanie Jed, Susan Kirkpatrick, et Kathryn Shevelow.

Lori Chamberlain a été professeure invitée au département de littérature de l'université de Californie, à San Diego. Elle est l'une des plus grandes théoriciennes ayant travaillé sur la traduction et le genre dans les années 1980, bien qu'après sa thèse, elle ne consacra qu'un seul article à la traductologie. Cet unique article, Gender and the metaphorics of translation fut publié en 1988 dans Signs et sa traduction est proposé ici. Il a bouleversé les translation studies aux États-Unis et la traductologie en France, en leur apportant une grille d'analyse féministe. Lori Chamberlain a également publié des articles sur des sujets variés comme la poésie ou la fiction contemporaines, mais aussi sur la pédagogie.

Chamberlain, L. 1988. " Gender and the Metaphorics of Translation » Signs: Journal of Women in Culture and Society 13, $454-472$.

Prairie, M.W., \& Chamberlain, L. 1994. « Due Process in the Accreditation Context », The Journal of college and university law 21, 61-109.

Chamberlain, L. 1989. «Bombs and Other Exciting Devices, Or The Problem of Teaching Irony », College English 51, 29-40.

Chamberlain, L. 1987. « The Subject in Exile: Puig's "Eternal Curse" on the Reader of These Pages ", Novel: A Forum on Fiction 20, 260. 
Chamberlain, L. 1985. « Ghostwriting the Text: Translation and the Poetics of Jack Spicer » Contemporary Literature 26, 426.

Chamberlain, L. 1982. Afterwords, Translation as Poetics in Postmodern Writing. Ph. D., Comparative Literature, University of California, Irvine.

1 Dans une lettre adressée à Joseph Joachim, violoniste du XIX ${ }^{\mathrm{e}}$ siècle, Clara Schumann déclare: «Bin ich auch nicht produzierend, so doch reproduzierend " (Même si je ne suis pas une artiste créative, je recrée) ${ }^{1}$. Bien qu'elle ait joué un rôle primordial dans la reproduction des œuvres de son époux, aussi bien comme concertiste que plus tardivement, pour en préparer l'édition, elle fut aussi une compositrice à part entière. Pourtant, jusqu'à une période récente, les historiens ${ }^{2}$ n'avaient prêté attention qu'à un seul compositeur de la famille. En effet, comme les études féministes l'ont amplement démontré, on trouve au fondement des représentations féminines traditionnelles qu'elles soient artistiques, sociales, économiques ou politiques - une ambivalence culturelle quant à la possible existence des femmes artistes et au statut de leurs «œuvres ». Dans le cas de Clara Schumann, l'ironie veut que ce soit le fait d'avoir eu à s'occuper des huit enfants conçus avec Robert Schumann qui l'empêcha d'être une compositrice plus productive.

2 Il nous semble que les déclarations selon lesquelles les femmes ne peuvent être de " grandes artistes » expriment un paradigme genré de répartition du pouvoir au sein de la famille et de l'État. Comme la recherche féministe dans différentes disciplines a pu le révéler, l'opposition entre le travail productif et reproductif organise la valeur qu'on donne au travail au sein d'une société : selon ce paradigme l'originalité et la créativité sont représentées en termes de paternité et d'autorité, reléguant la figure de la femme à une variété de rôles secondaires. Je m'intéresse à cette opposition spécifiquement parce qu'elle a toujours opéré une distinction entre écriture et traduction, opposant l'une, originale et masculine, à l'autre, dérivée et féminine. Si au premier abord cette distinction peut sembler n'être qu'une question esthétique, elle engendre toutefois d'importantes conséquences dans les domaines de la publication, du droit d'auteur, du cursus universitaire et de la titularisation. Ce que je propose ici, c'est d'examiner ce qui se joue au niveau du genre dans les représentations de la traduction: la lutte pour l'autorité ainsi que les politiques d'originalité qui sous-tendent cette lutte.

3 « Au mieux un écho $»^{3}$, la traduction a été représentée, littéralement et métaphoriquement, en des termes secondaires. On opère la même distinction qualitative entre l'acte original d'écriture et l'acte de traduction qu'entre l'acte original de composition musicale et son interprétation scénique par Clara Schumann. En effet, d'après les lois du copyright états-unien actuel, les traductions et les interprétations musicales sont traitées dans une seule et même rubrique dédiée aux "œuvres dérivées $»^{4}$. L'élaboration culturelle de cette perspective suggère que l'original renferme ce qui est naturel, vrai et légal, tandis que la copie détient ce qui est artificiel, faux et traître. Les traductions peuvent être, par exemple, des échos (lexique musical), des copies ou des portraits (lexique pictural), des vêtements empruntés ou mal-ajustés (lexique vestimentaire).

4 La sexualisation de la traduction la plus courante apparaît sans doute dans le lieu commun les belles infidèles ${ }^{5}$ : d'après cet aphorisme, les traductions devraient être, comme les femmes, soit belles, soit fidèles. En français, le cliché est rendu possible grâce à la rime et au fait que le mot traduction est un mot féminin, rendant ainsi la 
rime de l'expression masculine les beaux infidèles ${ }^{6}$ impossible. Ce lieu commun - forgé au $\mathrm{XVII}^{\mathrm{e}}$ siècle $^{7}$ - doit sa longévité à autre chose qu'à sa seule symétrie phonétique : c'est le fait d'avoir réussi à capter une complicité culturelle entre l'enjeu de la fidélité en traduction et celui de la fidélité dans le mariage qui lui donne l'apparence d'une vérité. Pour les belles infidèles, la fidélité est définie par un contrat implicite entre la traduction (en tant que femme) et l'original (en tant que mari, père, auteur). Cependant, l'infâme «deux poids, deux mesures » opère ici comme dans les mariages traditionnels : la femme/la traduction infidèle est jugée en place publique pour un crime que le mari/l'original est légalement en incapacité de commettre. En bref, ce contrat qui rend l'infidélité de l'original impossible est le révélateur d'une anxiété bien réelle face aux problèmes de la paternité et de la traduction : il imite le système de relations patrilinéaires où c'est la paternité - et non la maternité - qui rend une filiation légitime.

5 C'est cette lutte pour le droit de paternité, et le contrôle de la fidélité en traduction que celle-ci entraîne, que le comte de Roscommon explicite dans son traité du xvII siècle sur la traduction. Afin d'attester l'originalité du travail du traducteur, bien entendu nécessaire pour revendiquer une paternité, le traducteur doit usurper le rôle de l'auteur. Roscommon débute de façon plutôt bienveillante, en invitant le traducteur à "choisir l'auteur comme on choisit un ami». Mais cette intimité sert un objectif potentiellement subversif :

\begin{tabular}{|l|l|}
\hline Depuis ce lien sympathique qui & \\
vous unit & United by this Sympathetick \\
Grandissent parenté, amitié, & Bond, \\
affection & You grow Familiar, Intimate, and \\
Vos pensées, mots, styles, vos & Fond; \\
âmes sont à l'unisson, & Your thoughts, your Words, your \\
Et vous, plus juste son & Stiles, your Souls agree, \\
interprète, mais Lui. & Nolonger his Interpreter, but $\mathrm{He}^{8}$. \\
\hline
\end{tabular}

6 C'est un témoignage quasiment silencieux: à travers la familiarité (l'amitié), le traducteur devient, pour ainsi dire, membre de la famille et finalement le père luimême. Quelle que soit la bataille menée entre l'auteur et le traducteur, elle est dissimulée derrière le langage de l'amitié. Tandis que le traducteur est considéré comme un homme, le texte lui-même est considéré comme une femme dont la chasteté doit être préservée : 
Jeune muse est si facilement abusée

Qu'est précieux son honneur de pucelle!

Apparaît par vos soins, bons, précoces, paternels,

L'instruction chaste de ses fraîches années.

La première impression dans son cœur d'enfant

Sera la plus profonde et doit être la meilleure

Toute austérité nourrirait servile peur,

Blesserait pure oreille, tout son imprudent. »

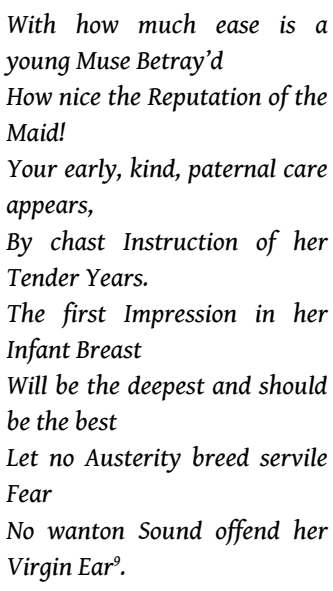

7 Alors que le traducteur devient l'auteur, il endosse certaines obligations paternelles vis-à-vis du texte, pour le protéger et l'instruire - ou peut-être pour le façonner. Le langage utilisé fait écho à celui des manuels de bonne conduite et reflète les différences attendues dans une éducation bienséante des hommes et des femmes: «une instruction chaste » convient à une femme, dont la virginité est un prérequis essentiel au mariage. Pour le texte, cette page blanche qui porte l'empreinte de l'auteur ( « La première impression [...] sera la plus profonde »), il est impossible d'être vierge par deux fois - la première pour l'auteur original, et l'autre pour le traducteur qui a pris sa place. C'est cette « chasteté » qui résout - ou refoule - la lutte pour la paternité ${ }^{10}$. Genrer la traduction par le biais d'un langage paternaliste est plus explicite chez Thomas Francklin, dans son traité du XVIII ${ }^{e}$ siècle sur la traduction.

\begin{tabular}{|l|l|}
\hline À moins qu'un auteur telle une & Unless an author like a \\
amante me réchauffe, & mistress warms, \\
Puis-je cacher ses défauts, goûter & How shall we hide his faults or \\
à ses charmes, & taste his charms, \\
Trouver chez lui toutes beautés, & How all his modest latent \\
chastes et cachées, & beauties find, \\
Retracer chaque trait plus & How trace each lovelier \\
charmant de son âme, & feature of the mind, \\
Chaque tache adoucir, chaque & Soften each blemish, and each \\
grâce rehausser, & grace improve, \\
Et lui rendre bel hommage & And treat him with the dignity \\
comme à tout être Aimé & \\
\hline
\end{tabular}

Comme le comte de Roscommon, Francklin représente le traducteur comme un homme qui usurpe le rôle de l'auteur, usurpation qui apparaît dans le genre grammatical et amène à un changement de sexe. Le traducteur est dépeint comme un séducteur; l'auteur, assimilé aux traits « féminins » de son texte, devient alors l'« amante » tandis qu'on force le pronom masculin à renvoyer aux attributs féminins du texte («Trouver chez lui toutes beautés, chastes ou cachées »). En introduisant une confusion entre le genre de l'auteur et le genre attribué au texte, Francklin «traduit » le rôle créatif de l'auteur par un rôle passif du texte, ce qui rend l'auteur relativement impuissant face au traducteur. Ce dernier fait de l'auteur-texte une amante flattée et séduite par ses 
intentions, et une collaboratrice toute disposée à se faire plus belle - et sans aucun doute, infidèle.

Cette belle infidèle, dont les imperfections ont été atténuées et les beautés par conséquent rehaussées, est dépeinte à la fois comme une maîtresse et comme un modèle vivant. En utilisant l'analogie populaire de la peinture, Francklin révèle également les codes genrés de cette convention mimétique du calque : le traducteur/ peintre doit séduire le texte afin de pouvoir « retracer » (traduire) les traits de son sujet. William Cowper donne une version plus élaborée de cette convention dans sa préface de l'Iliade d'Homère, bien qu'il défende un point de vue divergent sur la question de l'amélioration du texte original par la traduction: "Si un peintre qui prétend dessiner le portrait fidèle d'une belle femme en vient à lui donner plus ou moins de traits que ceux qui lui sont propres, ainsi qu'une forme générale de maintien de sa propre invention, il pourrait être taxé d'avoir produit un jeu d'esprit ${ }^{12}$, une curiosité à sa manière, mais en aucun cas la dame en question ${ }^{13}$. Cowper argumente en faveur d'une fidélité à la beauté du modèle, de peur que la traduction ne l'abaisse au rang d'un vulgaire « jeu d'esprit » ou, si on va plus loin dans la logique du texte, de peur de la rendre monstrueuse («lui donner plus ou moins de traits»). Pourtant, l'expression « la dame en question » renferme aussi l'idée qu'il s'agit de l'autre femme - la belle, et potentielle infidèle, maitresse. Quoi qu'il en soit, Cowper, comme le comte de Roscommon et Francklin, féminise le texte et rend l'homme traducteur/auteur responsable de sa réputation - c'est-à-dire de sa fidélité.

Le langage, comme les textes, est traditionnellement représenté en termes féminins : notre «langue maternelle ». Quand les débats esthétiques de la fin du XVIII siècle passent des problèmes de mimétisme à ceux de l'expression - ou selon la fameuse métaphore d'Abrams, du miroir à la lampe - les discussions sur la traduction leur emboîtent le pas. La relation du traducteur à la figure maternelle est soulignée par certains termes évoqués plus haut - fidélité et chasteté - et le problème fondamental persiste: trouver un moyen de réguler relations sexuelles (auctoriales) légitimes et progéniture.

12 Un exemple représentatif qui dépeint la traduction comme un problème de fidélité à la « langue maternelle » apparaît dans le travail de Schleiermacher dont le double intérêt pour la traduction et l'herméneutique a été déterminant dans l'élaboration d'une théorie de la traduction contemporaine. Au sujet de la conservation de l'altérité étrangère essentielle d'un texte en traduction, Schleiermacher soulève l'enjeu suivant : "Qui voudrait empêcher sa langue maternelle d'apparaître, autant que chaque genre le lui permette, sous son meilleur jour et de faire l'unanimité partout? Qui préférerait enfanter des bâtards plutôt que les portraits crachés de leurs parents? [...] Qui supporterait d'être accusé, comme ces parents qui abandonnent leurs enfants aux acrobates, de faire subir des distorsions étrangères et contre nature à sa langue maternelle au lieu de l'exercer dans une gymnastique qui lui est naturelle ${ }^{14}$ ?» Le traducteur, en tant que père, doit être fidèle à la mère/au langage afin de produire une descendance légitime; s'il tente d'enfanter autrement, il produira des bâtards tout juste bons pour le cirque. Parce que la langue maternelle est conçue comme naturelle, toute atteinte - toute infidélité - est perçue comme contre nature, impure, monstrueuse et immorale. Ainsi, c'est la loi «naturelle» qui réclame des relations monogames pour maintenir la "beauté » de la langue et pour assurer que les œuvres seront authentiques et originales. Bien que la référence de Schleiermacher à la 
bâtardise des enfants exprime une nette préoccupation de la pureté de la langue maternelle, il est aussi préoccupé de la paternité du texte. La « légitimité » a peu à voir avec la maternité, et davantage avec la reconnaissance institutionnelle de la paternité. La question: "Qui est le vrai père du texte?» semble alimenter cette double préoccupation de la fidélité de la traduction et de la pureté du langage.

Dans les métaphores de la traduction, la lutte pour les droits d'auteur a lieu à la fois dans la sphère familiale, comme nous avons pu le voir, et au niveau de l'État, car la traduction a aussi été représentée comme l'équivalent littéraire de la colonisation, une façon d'enrichir à la fois le langage et la littérature, nécessaire aux besoins politiques d'expansion des nations. La préface suivante, typique d'un traducteur britannique du XVIII ${ }^{\mathrm{e}}$ siècle, explicite cette idée: "Toi, mon Dieu, sais comme les œuvres de génie permettent à un pays de se hisser au premier rang des nations, et lui donnent autant d'honneur qu'un succès militaire. Parmi ces œuvres, nous devons rendre hommage à nos traductions des classiques grâce auxquelles, lorsque nous aurons naturalisé tant la Grèce que Rome, nous serons bien plus riches qu'elles de maintes productions originales, puisque nous avons déjà les nôtres ${ }^{15}$. » Le succès littéraire étant assimilé au succès militaire, la traduction a le pouvoir d'étendre tant les frontières littéraires que politiques. Les Romantiques allemands, qui utilisaient aussi bien le terme übersetzen (traduire) que verdeutschen (germaniser), percevaient l'entreprise de la traduction avec la même mentalité: pour eux, la traduction était littéralement une stratégie d'incorporation linguistique. Le grand modèle de cet usage de la traduction est de toute évidence l'Empire romain, qui s'incorpora la culture grecque de façon si spectaculaire. À propos des Romains, Nietzsche affirme : «traduire c'était conquérir ${ }^{16}$ ».

Il est à noter que les politiques de colonisation recoupent de façon significative les politiques du genre que nous avons rencontrées jusqu'à maintenant. Flora Amos montre, par exemple, qu'au xvi ${ }^{e}$ siècle en Angleterre, la traduction est perçue comme un « devoir public ». L'exemple le plus frappant de ce qu'on considère alors comme un « devoir public » est exposé par Thomas Drant, traducteur anglais d'Horace qui, dans la préface de sa traduction de l'auteur romain, proclame hardiment :

Tout d'abord, je lui ai fait ce qu'on avait commandé au peuple de Dieu de faire à leurs femmes captives qui étaient belles et ravissantes : je lui ai rasé la tête, coupé les ongles, c'est-à-dire que je lui ai arraché sa vanité, et sa superficialité matérielle. [...] J'ai anglicisé les éléments non pas dans la veine de la propriété latine, mais plutôt dans celle de sa propre langue vulgaire. [...] J'ai rapiécé sa logique, amplifié et soigné ses similitudes, adouci ses rudesses, prolongé toute forme étroite de discours, changé et beaucoup altéré ses mots, et non ses phrases, ou du moins (si j'ose dire) pas son objectif ${ }^{17}$.

Drant est en mesure de prendre les libertés qu'il décrit ici, puisque, en tant qu'ecclésiastique traduisant un auteur profane, on attend de lui qu'il rende Horace moralement acceptable: il doit le transformer considérablement pour faire de cet étranger ou cet autre un membre de la famille. En effet, le passage de la Bible auquel Drant fait allusion (Deutéronome 21 : 12-14) porte sur la bonne manière de faire d'une femme captive une épouse: "Tu pourras la prendre pour femme et l'amener en ta maison. Elle se rasera la tête, se coupera les ongles ${ }^{18}$. " Après lui avoir donné un mois pour faire son deuil, le ravisseur peut alors la prendre pour femme ; mais même s'il ne trouve en elle nul « délice », ce passage lui interdit ensuite de la vendre puisqu'il l'aura déjà humiliée. En faisant d'Horace une épouse acceptable, Drant doit le transformer en femme, fait troublant qui perdure dans la tension de la référence pronominale, où 
«lui » fait référence aux «femmes». De plus, la paraphrase de Drant donne la responsabilité de la tonte et des ongles à couper au mari-traducteur plutôt qu'à un Horace captif. Malheureusement, les ravisseurs faisaient souvent bien plus que raser la tête aux femmes captives (voir Nombres $31: 17-18$ ) ; on peut dresser un parallèle entre la violence sexuelle qui est évoquée dans cette description de la traduction et les viols politiques et économiques implicites dans une métaphore de la colonisation.

Il est clair qu'en traduction la signification du mot « fidélité » change en fonction de l'objectif poursuivi dans un contexte culturel et esthétique plus large. Dans sa version genrée, la fidélité définit parfois la relation de la traduction (femme) à l'original, en particulier à l'auteur original (homme), destitué et remplacé par l'auteur (homme) de la traduction. Dans ce cas, on doit protéger le texte, s'il est bon et beau, contre sa propension à l'infidélité afin d'authentifier l'originalité de cette production. Ou bien, la fidélité peut aussi définir une relation de l'auteur-traducteur (homme) à sa langue maternelle (femme), langue dans laquelle on est en train de traduire. Dans ce cas, la langue (femme) doit être protégée de la diffamation. C'est, de façon paradoxale, cette conception de la fidélité qui mène à justifier le viol et le pillage d'une autre langue et d'un autre texte, comme nous l'avons vu chez Drant. Mais là encore, ce type de fidélité est conçu pour enrichir la langue « d'accueil » en attestant l'originalité de la traduction : les conquêtes, faites captives, sont incorporées dans des "œuvres de génie » d'une langue donnée.

Il devrait désormais être évident que cette métaphore de la traduction révèle à la fois une anxiété face aux mythes de la paternité (ou du droit d'auteur et de l'autorité) et une ambivalence profonde vis-à-vis du rôle de la maternité - variant de la condamnation des belles infidèles à l'adulation vouée à la « langue maternelle ». Parmi les rares tentatives visant à gérer à la fois la pratique et l'allégorie de la traduction, Serge Gavronsky soutient que la source de cette anxiété et de cette ambivalence est tapie dans la structure œdipienne qui façonne les options du traducteur. Gavronsky divise le monde des métaphores de la traduction en deux camps. Il étiquette le premier groupe comme étant piétiste: les métaphores basées sur la coïncidence des conventions courtoises et chrétiennes, selon lesquelles le chevalier modèle fait vœu de fidélité à une dame immaculée, tel le chrétien à la Vierge. Dans ce cas, le traducteur (en tant que chevalier et chrétien) fait vœu d'humilité, de pauvreté - et de chasteté. En termes séculiers, cela est appelé traduction "positionnelle», puisque cela est basé sur une hiérarchisation bien connue des participants. À la relation verticale (auteur/ traducteur) s'ajoutent ainsi des implications métaphysiques et éthiques et, dans cette position du missionnaire, la soumission est proche de la dévotion.

Gavronsky soutient que le schéma maître/esclave inhérent à ce modèle métaphorique de la traduction est précisément au fondement du triangle œdipien :

Ici, en des termes typiquement euphémiques, l'esclave est consentant (un serviteur hyperbolique, un fidèle): le traducteur se considère comme l'enfant du pèrecréateur, son rival, tandis que le texte devient un objet de désir, entièrement défini par la figure paternelle, le stylo-phallus. Les traditions (les tabous) imposent au traducteur un rôle rituel hautement restreint. Il est obligé de s'équeuter (au sens littéral) afin de respecter l'interdiction de l'inceste. Altérer le texte reviendrait à éliminer, en tout ou en partie, le père-autor(ité), le dominant du moment ${ }^{19}$.

19 Ainsi, le «soin paternel» dont parle le comte de Roscommon est une des manifestations du refoulement de la relation incestueuse avec le texte, une autre étant la préoccupation pour la pureté des langues de la (Vierge) Mère. 
20 On peut voir l'autre côté du triangle œdipien comme un désir de tuer le père symbolique texte/auteur. D'après Gavronsky, l'alternative au traducteur piétiste est le traducteur cannibale " agressif qui s'empare de "l'original", qui savoure le texte, c'està-dire qui se nourrit vraiment de ses mots, qui les ingurgite et qui, ensuite, les prononce dans sa propre langue, en s'étant ainsi débarrassé du créateur "original" 20 ”. Tandis que le modèle piétiste présente les traducteurs comme tout à fait secondaires à ce qui est pur et original, Gavronsky déclare que le modèle "cannibale " libère les traducteurs de la servilité «à l'égard des restrictions idéologiques et culturelles». Ce que Gavronsky désire, c'est libérer le traducteur/la traduction des signes culturels d'infériorité, mais son modèle s'inscrit malheureusement dans le même ensemble de termes et logiques binaires que nous avons étudiés jusqu'ici dans les allégories de la traduction. En effet, nous pouvons voir dans la description suivante à quel point les métaphores de Gavronsky sont encore inscrites dans cette idéologie : «L'original a été capturé, violé, et l'inceste commis. Ici, une fois encore, l'enfant est le père de l'homme. L'original est mutilé jusqu'à en être méconnaissable ; la dialectique maître/esclave est

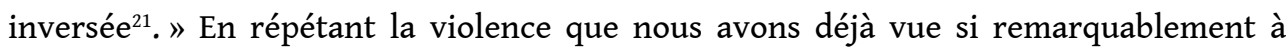
l'œuvre chez Drant, Gavronsky trahit les dynamiques de pouvoir dans ce système «paternel». Que le traducteur usurpe discrètement le rôle de l'auteur comme le préconise le comte Roscommon, ou qu'il s'empare de l'autorité à travers des moyens plus violents, le pouvoir est toujours représenté comme un privilège masculin exercé dans les sphères familiales et étatiques. Pour Gavronsky, le traducteur est un homme qui reproduit au niveau sexuel le même type de crimes que n'importe quel pays colonisateur commet sur ses colonies.

21 Ainsi que Gavronsky le reconnaît lui-même, la figure du traducteur cannibale s'appuie sur le modèle herméneutique de George Steiner, le théoricien contemporain de la traduction le plus éminent. L'influence du modèle de Steiner illustre la persistance, dans la théorie de la traduction contemporaine, de ce que j'ai appelé les politiques de l'originalité et ses logiques de violence. Dans Après Babel, Steiner propose de penser un déroulement de la traduction en quatre étapes. La première étape, celle de l'«élan initial de confiance », décrit l'empressement du traducteur à faire en toute confiance le pari que le texte lui cédera quelque chose. En guise de deuxième étape, le traducteur doit passer à un stade plus agressif en "pénétrant » et "capturant » le texte (Steiner appelle cela la "pénétration appropriative»), un acte explicitement comparé à la possession érotique. Durant la troisième étape, le texte emprisonné doit être "naturalisé », et doit intégrer la langue du traducteur, littéralement incorporé ou incarné. Enfin, pour compenser ce «ravissement appropriatif» le traducteur doit restaurer l'équilibre, et tenter un acte de compensation pour réparer cet acte d'agression. Son modèle pour cet acte de restitution dit-il, est « celui qui se dégage de l'Anthropologie structurale de Lévi-Strauss, et selon laquelle les structures sociales cherchent à rétablir l'équilibre à travers l'échange de mots, de femmes et de biens matériels ». Ainsi Steiner fait un lien explicite entre l'échange de femmes, par exemple, et l'échange de mots d'une langue contre les mots d'une autre ${ }^{22}$.

Steiner présente un argumentaire dont la politique sexuelle est explicite dans le chapitre introductif de son ouvrage, où il met en valeur le modèle d'une «lecture totale ». La traduction, en tant qu'acte d'interprétation, est un cas particulier de communication, et la communication est un acte sexuel : "Éros et le langage, point après point, forment un maillage inextricable. Rapport sexuel et rapport verbal, copule 
et copulation, sont des sous-classes du fait prédominant de la communication. [...] Le sexe est un acte profondément sémantique ${ }^{23}$." Steiner prend note d'une tendance culturelle à voir l'acte de communication du point de vue de l'homme et ainsi de valoriser la position du père/auteur/original, mais il reproduit lui-même en parallèle ce point de vue masculin, dont rend par exemple compte la description suivante de la relation entre le rapport sexuel et la communication: «On a souvent prouvé que la décharge sexuelle était plus puissante dans l'onanisme masculin que durant le rapport sexuel. J'incrimine comme facteur déterminant à l'œuvre dans ce phénomène une exceptionnelle souplesse dans l'expression et une vivacité de représentation hors du commun. [...] L'éjaculation est tout à la fois un concept linguistique et physiologique. L'impuissance et les blocages mutiques, l'éjaculation précoce et le bégaiement, l'éjaculation involontaire et le débit onirique des mots sont des phénomènes dont l'interrelation semble nous ramener au centre névralgique de notre humanité. Semence, excréments et mots sont les produits de la communication ${ }^{24}$. Ici, l'allusion à Lévi-Strauss, avec le passage déjà évoqué plus haut et qui apparaît plus tard dans le livre («l'échange de mots, de femmes, de biens»), offre un récit qui lie ensemble le discours, le rapport sexuel et la traduction, et qui le fait du point de vue d'un traducteur masculin. En effet, nous remarquons que lorsque la communication est en jeu, ce qui peut être échangé est dépeint au moins partiellement en termes masculins (" sperme, excréments, et les mots »), tandis que lorsque la « restitution » est en jeu, ce qui peut être échangé est dépeint en termes féminins.

En écrivant conformément au système hiérarchisé du genre, Steiner semble plus loin défendre l'idée que le paradigme est universel et que les rôles masculins et féminins qu'il décrit sont essentiels plutôt qu'accidentels. D'un autre côté, il remarque que les règles des rapports verbaux (et probablement, celles des rapports sexuels) sont sociales, et il souligne les différences langagières que cela entraîne entre hommes et femmes.

À première vue, la parole des femmes abonde, plus que celle des hommes, de nuances dans l'expression du désir et de l'avenir que le grec et le sanskrit conjuguent à l'optatif. L'éventail verbal des femmes permet plus de prudence et dissimulation dans l'expression des décisions et des promesses. [...] Je ne prétends pas qu'elles déforment la toile étriquée et réfractaire du monde, mais elles agrémentent la réalité d'une multiplicité de facettes, renforcent l'adjectif jusqu'à lui offrir la possibilité d'un statut nominal, d'une manière que les hommes trouvent déstabilisante. On trouve dans l'intonation masculine du pronom à la première personne le couperet de l'ultimatum, une attitude séparatiste. Le « Je » des femmes suggère quant à lui une attitude plus patiente, ou du moins était-ce le cas jusqu'aux mobilisations des femmes pour leur libération. Les deux modèles de langage s'inspirent de l'aphorisme de Robert Graves selon lequel les hommes « font » et les femmes « sont $»^{25}$.

Mais, tout en reconnaissant les forces sociales et économiques à l'œuvre dans ces différences, il veut aussi croire en une cause biologique fondamentale: "Pourtant certaines différences linguistiques laissent penser qu'elles ont un fondement physiologique, ou plus précisément, un fondement à la limite du biologique et du social ${ }^{26}$. " Steiner se garde bien d'insister sur leurs prémisses biologiques, mais il y a dans sa propre rhétorique une tendance à considérer comme immuables jusqu'aux différences de socialisation langagière entre hommes et femmes. Si le fondement sexuel de la communication comme fondement de la traduction doit être considéré comme universel, alors Steiner semble s'inscrire fermement dans la tradition que nous avons 
examinée, selon laquelle «les hommes font» tandis que "les femmes sont». Cette tradition ne se limite pas, bien entendu, au domaine de la traductologie. Aussi l'influence de Steiner et Lévi-Strauss est telle qu'il n'est pas surprenant que le genre soit devenu la clef de voûte de la communication dans des domaines voisins comme la sémiotique ou la critique littéraire ${ }^{27}$.

Comme nous l'avons démontré jusqu'ici, l'allégorie de la traduction est symptomatique d'enjeux plus vastes de la culture occidentale : d'une répartition du pouvoir fondée sur le genre dans les relations; d'un désir obstiné (sans être toujours hégémonique) de faire coïncider le langage ou son usage avec la moralité ; d'une quête d'authenticité ou d'unité et, par conséquent, d'une intolérance à la duplicité, à l'impossibilité de trancher. Voici la question fondamentale : pourquoi a-t-on associé métaphoriquement ces deux univers de la traduction et du genre ? Si on reprend les termes d'Umberto Eco, quelle est la codification métonymique ou le récit sous-jacent à ces deux domaines ${ }^{28}$ ?

Notre analyse des métaphores de la traduction semble suggérer le récit implicite d'un combat au mérite entre production et reproduction. L'éternel enjeu de ce qui se présente comme un problème esthétique, et passe par les termes de sexe, de famille et d'État, est le pouvoir. Plus haut, nous avons pu voir comment le concept de fidélité permet de contrôler la sexualité et/dans la famille afin de garantir que l'enfant soit la production du père, reproduit par la mère. Ce contrôle est signe d'autorité et de pouvoir paternel, une façon de manifester la paternité de l'enfant - qui est par ailleurs une sorte de fiction - et ainsi de réclamer l'enfant comme sa progéniture légitime. Ceci n'est donc évidemment pas sans lien avec la possession et le legs de propriété. Dans le mariage, comme en traduction, il y a une dimension légale au concept de fidélité. Par exemple, il n'est pas légal (devrais-je dire légitime?) de publier la traduction de travaux qui ne sont pas encore dans le domaine public, sans l'accord de leur auteur (ou des ayants droit). Il est d'usage d'établir un contrat en bonne et due forme avant d'annoncer la naissance d'une traduction, ceci afin d'en clarifier les liens de parenté. D'après les codes liés à la division du travail, et établis pour un marché qui privilégie l'activité masculine et la rétribue en conséquence, on donne plus de valeur à la production qu'à la reproduction. La transformation de la traduction d'une activité reproductive en une activité productive, d'une œuvre secondaire en une œuvre originale, reprogramme les droits de traduction en droits de propriété - signes de richesse, signes de pouvoir.

J'irai plus loin en affirmant que si la traduction est ultra codifiée et ultra réglementée à ce point, c'est qu'elle menace d'effacer la différence entre production et reproduction, essentielle à l'assise du pouvoir. En bref, les traductions, travesties en originaux, ont le pouvoir de court-circuiter le système. Cette différence est maintenue coûte que coûte : "Tout lecteur déçu sait qu'un poème risque sa vie à chaque traduction..$^{29}$ "

Le danger posé par l'infidélité est présenté ici comme une question de vie ou de mort ; dans un commentaire sur les traductions des classiques de la Loeb Library, Rolfe Humphries évoque ce risque en termes plus vivaces: "Elles émasculent leurs originaux $^{30}$.» La violence sexuelle, implicite dans la métaphore de Drant sur la traduction, peut être comprise comme une violence qui s'exerce non seulement à l'encontre de l'essence féminine du texte («femmes captives») mais aussi du signe de l'autorité masculine. Car, comme l'histoire de Samson et de Dalila nous l'apprend, la tonsure à laquelle se livre Drant ( "Je lui ai rasé la tête, coupé les ongles, c'est-à-dire que je lui ai arraché sa vanité, et sa superficialité matérielle.») peut suggérer la perte 
du pouvoir masculin, une castration symbolique. C'est ce qu'un critique appelle le manque inévitable ${ }^{31}$ : ce que l'original risque de perdre, en bref, c'est son phallus, le signe de la paternité, d'autorité, et d'originalitééc

Dans le système métaphorique que nous examinons ici, l'objet revendiqué par le traducteur, c'est précisément le droit à la paternité. Dans une codification patriarcale, revendiquer le phallus est le seul moyen qui lui reste pour revendiquer sa légitimité vis-à-vis du texte. Défendre l'idée que traduire c'est comme écrire est une façon de faire d'une simple activité re-créative une activité créative. Mais les revendications d'originalité et d'autorité, formulées en référence à des actes de création artistique et biologique, contrastent fortement avec la place de la traduction dans une hiérarchie littéraire ou économique. En effet, tandis que l'écriture et la traduction partagent sans doute les mêmes représentations de division des sexes et du pouvoir - une préoccupation vis-à-vis du droit d'auteur et de l'autorité - la traduction ne partage pas les mythes rédempteurs de noblesse ou de triomphe que nous associons à l'écriture. Ainsi, malgré leurs revendications métaphoriques pour être considérés à l'égal des écrivains, les traducteurs sont souvent honnis ou ignorés : dans les grands journaux, il n'est pas rare de tomber sur des critiques de traduction qui ne mentionnent ni le traducteur, ni le processus de traduction. Aujourd'hui encore à l'université, considérer un projet de traduction comme digne d'un sujet de thèse ou d'un dossier de titularisation reste un fait marginal, hormis dans le cas où la renommée de l'auteur est suffisante pour qu'un projet soit accepté. Malgré le travail d'organisations et associations, comme PEN ou ALTA, pour l'amélioration des conditions économiques, l'aide à la gestion, la défense des droits et le conseil juridique, même les meilleur'es traducteurs et traductrices ne gagnent encore que des salaires dérisoires. Ce mépris absolu de la part du monde universitaire contraste vivement avec son recours systématique à la traduction pour l'étude des "classiques » de la littérature mondiale, des grands textes critiques et philosophiques, et des chefs-d'œuvre jusque-là méconnus $\mathrm{du}$ "Tiers »- monde. Tandis que les métaphores que nous avons étudiées cherchent à camoufler le statut secondaire de la traduction derrière un langage phallique, la culture occidentale renforce cette secondarité de façon vindicative, en insistant sur le statut féminisé de la traduction. Ainsi, bien qu'on trouve assurément des hommes comme des femmes en traduction, la logique binaire qui nous encourage à définir les infirmières comme des femmes et les médecins comme des hommes, les enseignantes comme des femmes et les professeurs comme des hommes, les secrétaires comme des femmes et les cadres d'entreprise comme des hommes, définit également la traduction, à bien des égards, comme un archétype de l'activité féminine.

Même lorsque les termes de la comparaison sont inversés pour souligner le caractère re-créatif des deux activités - lorsque l'on dit qu'écrire c'est comme traduire, il est intéressant de voir, là aussi, que le biais de genre ne disparaît pas. Par exemple, dans son court essai qui examine la relation entre la traduction et certains courants de la théorie critique contemporaine, Terry Eagleton défend l'idée suivante :

Il se pourrait alors que la traduction d'une langue vers une autre puisse exposer à nos yeux quelque chose des mécanismes productifs mêmes de la textualité ellemême. [...] Les théories critiques excentriques mais suggestives d'Harold Bloom [...] soutiennent que chaque créateur poétique est emprisonné dans une rivalité œdipienne avec un précurseur patriarcal « fort » - que cette " création » littéraire [...] est en réalité une question de lutte, d'anxiété, d'agression, d'envie et de répression. Même si cela est embarrassant, le «créateur » ne peut invalider le fait que [...] son poème se tapit dans l'ombre d'un poème ou d'une tradition poétique 
antérieure, dont il doit combattre l'autorité pour trouver sa propre " autonomie ». Dans la lecture de Bloom, tous les poèmes sont des traductions, ou des «erreurs d'interprétation créatives " d'autrui ; et le traducteur littéral seulement, peut-être, est celui qui connaît le plus ardemment le coût psychique et l'enchantement que requiert tout acte d'écriture ${ }^{33}$.

31 Ce qu'Eagleton essaie de nous faire comprendre à travers les propos de Bloom, c'est que le mécanisme créatif et productif de l'écriture n'est pas original, c'est-à-dire que les textes n'émergent pas ex nihilo; au contraire, tant l'écriture que la traduction dépendent de textes précédents. Renversant la hiérarchie traditionnelle, il évoque le statut secondaire de la traduction comme un modèle pour l'écriture. Cependant, en mettant sur le même plan traduction et "erreurs de lecture ", Eagleton (à travers Bloom) leur trouve un dénominateur commun dans la lutte contre un "précurseur patriarcal puissant»: le mécanisme productif et créatif est, encore une fois, entièrement masculin. La tentative d'Eagleton comme celle de Bloom de remplacer le concept d'originalité par le concept de traduction ou d'erreur de lecture créatives est un tour de passe-passe. Chez eux, le genre et les métaphores de la traduction ne font que changer d'appellation, puisque le concept de traduction reste défini avec les mêmes termes patriarcaux dont nous avons observé l'usage dans les définitions de l'originalité et de la production.

Cependant, dans le même temps, une part importante de la nouvelle théorie critique a aussi remis en question les mythes de l'autorité et de l'originalité qui font de l'écriture une activité masculine supérieure à la traduction. Les théories de l'intertextualité, par exemple, rendent difficile la démarcation des frontières précises du texte, et gomment, par conséquent, l'idée d'« origines »: le texte n'est plus simplement le produit d'un individu (mâle ?) autonome, mais trouve ses sources dans l'histoire, c'est-à-dire au sein de codifications sociales et littéraires articulées par un·e auteur·e. Les études féministes ont porté à notre attention un corpus considérable d'écrits produits par des femmes, des écrits jusque-là marginalisés ou censurés par le canon universitaire; ainsi, ces études mettent en lumière le conflit entre les théories de l'écriture codées comme masculines et la réalité de l'auteurice. Ces études, autour du rôle joué par le genre dans nos concepts d'écriture et de production, nous forcent à réexaminer les hiérarchies qui ont subordonné la traduction à un concept d'originalité. La réinterprétation de la traduction qui en résulte a bien sûr des conséquences pour tout type d'activités liées au travail interprétatif, car la traduction a été utilisée comme une métaphore ou un modèle conventionnel pour toute une variété d'actes de lecture, d'écriture, et d'interprétation. En effet, il serait profitable d'analyser l'analogie entre traduction et interprétation à travers le prisme du genre, car son usage dans ces discours contredit probablement l'idée d'enjeux pourtant similaires à propos de l'autorité, de la violence, et du pouvoir.

La théorie réformiste de la traduction la plus influente est celle de Jacques Derrida dont le projet a été de subvertir le concept même de différence qui produit l'opposition binaire entre l'original et sa reproduction, pour finalement rendre cette différence indéterminable. En mobilisant plusieurs termes du champ lexical de la différence sexuelle - dissémination, invagination, hymen - Derrida expose le genre comme un cadre conceptuel pour les définitions de la mimesis et de la fidélité, définitions centrales d'une vision "classique " de la traduction. Le problème de la traduction, implicite dans tout son travail, est devenu de plus en plus explicite depuis son essai «LIVING ON. Border Lines ${ }^{34}$ », dont les prétextes sont le « Triumph of Life » de Shelley 
et L'Arrêt de mort de Blanchot. En suggérant «l'inter-traduisibilité » de ces textes, il défie les conventions non seulement de la traduction mais également de l'ascendance et de l'auctorialité.

Cet essai parle de traduction, à plusieurs titres. Tout d'abord, il parut en anglais c'est-à-dire, grâce à sa traduction. Par ailleurs, on y trouve une note de bas de page fleuve de l'auteur sur les difficultés rencontrées lorsqu'il s'est agi de traduire ses propres termes, ambigus, ainsi que ceux de Shelley et de Blanchot. Il expose au passage l'impossibilité du " rêve de traduction sans résidus ${ }^{35}$ ». Selon lui, il y a toujours quelque chose qu'on abandonne et qui trouble les distinctions entre original et traduction. Il n'y a pas de traduction "silencieuse ». Par exemple, il note l'importance des mots écrit, récit, et série dans le texte de Blanchot et demande : "Note aux traducteurs : Comment allez-vous traduire cela, récit, par exemple ? Pas comme nouvelle ${ }^{36}$, "novella", ni comme "short story". Peut-être que le mieux serait de laisser le terme "français" récit. Il est déjà assez difficile de comprendre ce terme dans le texte français de Blanchot ${ }^{37}$. "

L'impossibilité de traduire un mot comme récit est, d'après Derrida, une fonction de la loi de la traduction, et non une question d'infidélité ou de secondarité de la traduction. La traduction est gouvernée par la double contrainte de l'exigence « Ne me lisez pas »: le texte tout à la fois exige et prohibe sa traduction. Derrida parle de cette double contrainte de la traduction comme d'un hymen, à la fois signe de virginité et de consommation du mariage. Ainsi, en tentant de renverser les oppositions binaires déjà étudiées plus haut dans d'autres analyses, Derrida sous-entend que la traduction est à la fois originale et secondaire, intacte et transgressée ou transgressive. Reconnaissant également que le traducteur est souvent une femme - si bien que, souvent, son sexe et la secondarité genrée attribuée à la tâche coïncident - Derrida, dans L'Oreille de l'autre, continue à défendre l'idée que «la femme traductrice dans ce cas-là n'est pas simplement subordonnée, ce n'est pas la secrétaire de l'auteur, c'est aussi celle qui est aimée de l'auteur et à partir de laquelle seule, l'écriture est possible. La traduction est une écriture, n'est pas simplement une traduction au sens de la transcription, c'est une écriture productive qui est appelée par le texte original ${ }^{38}$. » En défendant l'idée d'une interdépendance de l'écriture et de la traduction, Derrida subvertit l'autonomie et le privilège du texte "original ", en l'attachant à un contrat impossible mais nécessaire avec la traduction et en les rendant redevables l'un envers l'autre.

En mettant l'accent à la fois sur les aspects reproductifs et productifs de la traduction, le projet de Derrida - et, curieusement, la traduction de son travail - fournit les conditions pour une exploration nécessaire des contradictions de la traduction et du genre. Précédemment, son travail avait déjà donné naissance à un recueil d'essais avec la traduction comme point de départ pour parler de philosophie, d'interprétation et d'histoire littéraire ${ }^{39}$. Ces essais, bien qu'ils n'abordent pas de manière explicite la question du genre, s'appuient sur son idée de duplicité de la traduction sans l'idéaliser ou l'assujettir aux termes qui sont conventionnellement dominants. Dans son propre travail, Derrida n'attache pas vraiment d'importance aux circonstances historiques ou culturelles de textes spécifiques, circonstances qui ne peuvent pourtant pas être ignorées dans l'investigation des problématiques de la traduction ${ }^{40}$. Par exemple, à une certaine époque, les femmes avaient le droit de traduire, précisément parce que cela était considéré comme une activité secondaire ${ }^{41}$. Notre tâche en tant qu'universitaires, c'est d'apprendre à écouter le discours « silencieux » - des femmes, en traductrices - 
afin de mieux démêler les liens existant entre ce qui a été codifié comme un discours «faisant autorité » et ce qui est tu par peur de la disruption ou de la subversion.

Au-delà de ce type de connaissances, une théorie féministe de la traduction nécessite une pratique gouvernée par ce que Derrida appelle la double contrainte - et non le " deux poids, deux mesures ». Une telle théorie ne peut pas s'appuyer sur le modèle familial de la lutte œdipienne, mais doit marcher sur le fil du rasoir de la traduction comme collaboration, où l'auteur et le traducteur sont vus comme des collaborateurs, tant sur le plan de la coopération que de la subversion. Ce modèle répond à des problèmes exprimés par un nombre croissant de femmes traductrices qui commencent à se demander, comme Suzanne Jill Levine, ce que cela veut dire d'être une femme traductrice dans et d'une culture masculine. En parlant plus spécifiquement de sa traduction récente de La Habana para un infante difunto de Cabrera Infante, un texte qui "se moque des femmes et de leurs mots ", elle pose la question suivante : "Que reste-til à une femme qui traduit ce genre de livre ? Ne devient-elle pas une double traîtresse, en se faisant l'Écho de ce Narcisse, en répétant l'archétype une énième fois? Toutes celles qui utilisent la langue paternelle de la mère, qui se font l'écho des idées et des discours des grands hommes, deviennent en un sens des traîtresses: c'est la contradiction et le compromis de la dissidence ${ }^{42}$." Le choix même des textes sur lesquels travailler pose alors un dilemme initial pour la traductrice féministe: bien qu'un texte tel que celui de Cabrera Infante soit choquant idéologiquement, ne pas le traduire reviendrait à capituler devant cette logique qui attribue tout le pouvoir à l'original. Levine choisit plutôt de subvertir le texte, de faire jouer l'infidélité contre l'infidélité, et de suivre jusqu'au bout la logique parodique de l'ouvrage. Carole Maier, en examinant les contradictions à l'œuvre dans son rapport au poète cubain Octavio Armand, fait une remarque similaire en défendant l'idée que " la quête du traducteur n'est pas de faire taire mais de faire entendre, de rendre disponibles des textes qui soulèvent des questions difficiles et qui ouvrent des perspectives. Il est essentiel qu'en tant que traductrices les femmes se glissent aussi bien dans la peau de travaux antagonistes que favorables. Elles doivent devenir indépendantes, "résister" aux interprètes qui non seulement laissent parler les travaux des adversaires [...] mais aussi parlent avec eux et les replace dans un contexte plus large en les analysant, eux et leurs processus de traduction ${ }^{43}$." Son essai raconte son combat pour traduire une poésie d'Armand qui réduit sa propre mère au silence, et sa capacité à "résister " à l'injonction au silence qui lui est faite en tant que traductrice, pour donner voix aux contradictions du travail d'Armand. En refusant de réprimer sa propre voix lorsqu'elle parle pour la voix du "maître », Maier, tout comme Levine, parle à travers et contre la traduction. Les travaux de ces deux traductrices illustrent non seulement l'importance de la traduction mais aussi celle d'écrire à son sujet, en intégrant les principes d'une pratique au dialogue autour de la révision des traductions. C'est seulement à partir du moment où les femmes traductrices commenceront à discuter de leur travail - et quand assez de travaux historiques sur les traductrices jusque-là réduites au silence auront été produits - que nous serons capables de dessiner des alternatives aux luttes œdipiennes pour les droits d'auteur.

Les féministes qui travaillent sur la traduction font face à un champ presque ou totalement inexploré. Nous pouvons, par exemple, examiner : le rôle historique de la traduction dans l'écriture des femmes de différentes périodes et cultures; les problèmes particuliers posés par la traduction de textes explicitement féministes, comme par exemple ceux que discute Myriam Diaz-Diocaretz à propos de la traduction 
d'Adrienne Rich en espagnol ${ }^{44}$; les effets du canon et du marché sur le choix des textes à faire traduire, et par qui, des traducteur.rices, et des modalités de marchandisation ; les effets des traductions sur le canon et le genre; le rôle des formes d'écriture "silencieuses " comme la traduction qui redonnent voix aux discours de femmes et subvertissent les formes d'expression hégémoniques. Les théories féministes et poststructuralistes nous ont encouragées à lire entre les lignes, ou en dehors, des discours dominants pour comprendre les structures et l'autorité culturelles. La traduction peut fournir une source inépuisable d'informations sur les pratiques de domination et de subversion. De plus, comme les commentaires de Levine et Maier nous l'indiquent, un des défis pour les traductrices féministes serait de dépasser ces questions de sexe de l'auteuree ou du/de la traducteurrice. En travaillant au sein des hiérarchies conventionnelles déjà étudiées ici, la traductrice d'une autrice et le traducteur d'un auteur seront liées par les mêmes relations de pouvoir : ce qui doit être subverti, c'est le processus par lequel la traduction se conforme aux constructions du genre. Dans cette perspective, une théorie féministe de la traduction sera enfin utopique. Si les femmes écrivent leurs propres métaphores de la production culturelle, il devient alors possible de considérer les actes de rédaction, de création ou de légitimation des textes en dehors des binarités genrées qui ont fait des femmes, comme des traductions, les maîtresses de travaux proches de ceux qui éloignèrent Clara Schumann de ses compositions.

\section{NOTES}

1. NdT : Notre interprétation de la phrase originale serait: "Je ne suis pas non plus celle qui produit, mais après-tout je reproduis", mais, il nous a semblé important de garder ici l'interprétation de Lori Chamberlain qui introduit le concept « d'artiste créative » : « Even if I am not a creative artist, still I am recreating ".

Joachim, Joseph. 1911-13. Briefe von und an Joseph Joachim, éd. Johannes Joachim et Andreas Moser, 3 vols. Berlin : Julius Bard, vol. 2 : 86 ; cité dans Reich, Nancy B. 1985. Clara Schumann: The Artist and the Woman. Ithaca, N.Y.: Cornell University : 320 ; la traduction [en anglais était de] Reich. Voir le chapitre intitulé « Clara Schumann Composer and Editor » : 225-257.

2. NdT : Le masculin universel a été conservé lorsqu'il semblait désigner le fait d'hommes, ou des pratiques menées au détriment des femmes.

3. Il s'agit du titre d'un essai de Pires, Armando S. 1952. Americas 4(9) : 13-15, cité dans Brower, Reuben A. (éd.). 1959. On Translation. Cambridge, Mass. : Harvard University Press : 289.

4. United States Code Annotated, Title 17, Sect. 101. St. Paul, Minn. : West Publishing Co., 1977

5. NdT : En français dans le texte.

6. $\mathrm{NdT}$ : En français dans le texte.

7. Zuber, Roger. 1968. Les « Belles Infidèles » et la formation du goût classique. Paris : Librairie Armand Colin : 195 .

8. Comte de Roscommon. [1975]. "An Essay on Translated Verse" in English Translation Theory-1650-1800, T. R. Steiner (éd.). Amsterdam : Van Gorcum, Assen : 77.

9. Ibid. : 78. 
10. Sur la femme comme " une page blanche », voir Gubar, Susan. 1982. " "The Blank Page" and Issues of Female Creativity" in Writing and Sexual Difference, Elizabeth Abel (éd.). Chicago: University of Chicago Press : 73-94. Voir aussi Jed, Stephanie. 1989. Chaste Thinking: The Rape of Lucretia and the Birth of Humanism. Bloomington : Indiana University Press.

11. Thomas Francklin. [1975]. «Translation: A Poem » in English Translation Theory-1650-1800, T. R. Steiner (éd.). Amsterdam : Van Gorcum, Assen : 113-114.

12. NdT : En français dans le texte.

13. William Cowper. [1975]. «Preface to The Iliad of Homer » in English Translation Theory-1650-1800,

T. R. Steiner (éd.). Amsterdam : Van Gorcum, Assen : 135-136.

$\mathrm{NdT}$ : Notre traduction en français ici.

14. Schleiermacher, Friedrich. 1977. "Uber die verschiedenen Methoden des Ueber- setzen» traduction anglaise Andre Lefevere, in Translating Literature: The German Tradition from Luther to Rosenzweig, éd. Andre Lefevere. Amsterdam : Van Gorcum, Assen : 79.

$\mathrm{NdT}$ : Notre traduction en français ici.

15. Cité dans Amos, Flora Ross. [1920] 1973. Early Theories of Translation. New York: Octagon Books : 138-139. NdT : Notre traduction en français ici.

16. Nietzsche, Friedrich [1974]. The Gay Science. Traduction anglaise de Walter Kauffmann. New York : Random House : 90.

NdT : Notre traduction à partir de celle de Patrick Wotling de 1997, rééditée en 2020 : Nietzsche, Friedrich. 2020. Le Gai Savoir. Flammarion, coll. Prépas scientifiques 2021 : Paris. Edition Kindle : paragraphe 83 , livre second (« on se livrait à une conquête lorsqu'on traduisait »).

17. Cité dans Amos Ibid. : 112-113. NdT : Notre traduction en français ici.

18. La Bible de Jérusalem, Editions du Cerf, 1955.

19. Gavronsky, Serge. 1977. «The Translator: From Piety to Cannibalism » SubStance $16: 53-62$, en particulier 55. NdT : Notre traduction en français ici.

20. Ibid., 60.

21. Ibid.

22. Steiner, George. 1975. After Babel. Londres : Oxford University Press : 296, 298, 300, 302. NdT : Notre traduction ici. Lucienne Lotringer a publié en 1998 une excellente traduction de l'ouvrage de George Steiner : Après Babel : Une poétique du dire et de la traduction. Paris : Editions Albin Michel. Mais afin de rendre compte de la logique de Chamberlain qui critique les images sexualisées utilisées par Georges Steiner nous avons préféré garder des traductions plus calquées sur la version anglaise originale utilisée par l'autrice.

23. Ibid. : 38. $\mathrm{NdT}$ : Notre traduction ici.

24. Ibid. : 44, 39. NdT : Notre traduction ici.

25. Ibid. : 41. NdT : Notre traduction ici.

26. Ibid. : 43. NdT : Notre traduction ici.

27. Dans la critique acerbe de la sémiotique de Christine Brooke-Rose dont il est question ici, on retrouve la même analyse de l'usage de Levi-Strauss par Steiner : cf. Brooke-Rose, Christine.1985. «Woman as Semiotic Object» Poetics Today 6(1-2) : 9-20; ré-imprimé en 1986 par Susan Rubin Suleiman (éd.) The Female Body in Western Culture: Contemporary Perspectives, Cambridge, Mass. : Harvard University Press : 305-316.

28. Eco, Umberto. 1979. The Role of the Reader: Explorations in the Semiotics of Texts. Bloomington: Indiana University Press : 68.

29. Matthews, Jackson. 1959. "Third Thoughts on Translating Poetry ", in Brower, Reuben A. (éd.). On Translation. Cambridge, Mass. : Harvard University Press : 69.

30. Humphries, Rolfe, 1959. "Latin and English Verse - some Practical Considerations", in Brower, Reuben A. (éd.). On Translation. Cambridge, Mass. : Harvard University Press : 65.

31. $\mathrm{NdT}$ : En français dans le texte. 
32. Lewis, Philip. 1981. "Vers la traduction abusive ", in Les fins de l'homme : à partir du travail de Jacques Derrida, Philippe Lacoue-Labarthe et Jean-Luc Nancy (éds.). Paris: Editions Galilée: 253-261, en particulier 255.

33. Eagleton, Terry. 1977. «Translation and Transformation " Stand 19(3) : 72-77, en particulier 73-74.

34. Derrida, Jacques. 1979. «Living On / Border Lines" traduit en anglais par James Hulbert, in Deconstruction and Criticism, Harold Bloom et al. (éds.). New York : Seabury Press : 75-176.

NdT : Paru plus tard en français, sous le titre de «Survivre », et à nouveau en anglais sous le titre de «Living On ", il fut à l'origine édité en anglais dans Deconstruction and Criticism, New York, Continuum Intl Pub Group, 1995, sous le titre LIVING ON. Border Lines grâce à une traduction de James Hulbert.

35. $\mathrm{NdT}$ : Notre traduction ici.

36. $\mathrm{NdT}$ : En français dans le texte.

37. Ibid. 119, 86. $\mathrm{NdT}$ : une traduction possible serait «narrative » comme pour la traduction de Temps et Récit de Paul Ricoeur par Kathleen McLaughlin, David Pellauer : Time and Narrative. T. 1, 2, 3. Chicago : University of Chicago Press. 1990.

38. Ibid., 145 ; Derrida, Jacques. 1985. The Ear of the Other: Otobiography, Transference, Translation, McDonald, Christie V. (éd.), traduction anglaise de Peggy Kamuf. New York: Schocken Books : 153. $\mathrm{NdT}$ : Ici nous avons repris l'extrait dans la transcription de Lévesque, Claude et McDonald, Christie V. (dir.). 1982. L'oreille de l'autre. Otobiographies, transferts, traductions. Textes et débats avec Jacques Derrida. Montréal : VLB éditeur.

39. Graham, Joseph F. (éd.). 1985. Difference in Translation. Ithaca, N.Y. : Cornell University Press.

40. Pour une critique de «Living On / Border Lines » de Derrida, proche de la nôtre, voir l'essai de Mehlman, Jeffrey. 1984. « Deconstruction, Literature, History: The Case of L'Arret de mort » in Literary History: Theory and Practice, Sussman, Herbert L. (éd.). Boston : Actes du Northeastern University Center for Literary Studies, et Mehlman, Jeffrey. 1986. «Writing and Deference: The Politics of Literary Adulation », Representations 15 : 1-14.

41. Hannay, Margaret P. (éd.). 1985. Silent But for the Word: Tudor Women as Patrons, Translators, and Writers of Religious Works. Kent, Ohio : Kent State University Press.

42. Levine, Suzanne Jill. 1984. "Translation as (Sub)Version: On Translating Infante's Inferno » SubStance $42: 92$.

43. Maier, Carol. 1985. "A Woman in Translation, Reflecting " Translation Review 17 : 4-8, en particulier. 4.

44. Diaz-Diocaretz, Myriam. 1985. Translating Poetic Discourse: Questions on Feminist Strategies in Adrienne Rich. Amsterdam/Philadelphia : John Benjamins Publishing Co. Pour d'autres ouvrages qui répondent spécifiquement au problème du genre et de la traduction, voir aussi le numéro spécial de Translation Review on women in translation, 17 (1985); et Christ, Ronald. 1980. "The Translator's Voice: An Interview with Helen R. Lane » Translation Review 5 : 6-17.

\section{RÉSUMÉS}

En 1988, dans « Gender and the Metaphorics of Translation », Chamberlain revient sur la figure de la traduction à travers plusieurs siècles de métaphores en traductologie. À partir de l'étude de textes et recueils allant de 1958 à 1985, comme ceux de Serge Steiner ou Serge Gavronsky - et de 
ceux qui les ont inspirés depuis 1684, comme Earl of Roscommon ou William Cowper - elle s'appuie sur Jacques Derrida, Terry Eagleton, Joseph Graham, Carole Maier, Suzanne Jill Levine ou Susan Gubar, en approfondissant leur approche, pour analyser à la fois le statut subalterne donné par les grands traductologues à la traduction face à l'écriture, mais aussi le rapport sexualisé, soumis ou dominateur, que celle-ci est sensée entretenir avec le texte original. Cette analyse rédigée en plein essor de la traductologie féministe outre-Atlantique remet en cause une conception masculine et sexiste de la traduction et de la créativité décrites en termes de domination, de pouvoir, de sexe et de violence. En faisant tomber le masque séducteur de la beauté stylistique des textes de traductologues comme Steiner ou Gavronsky, elle nous rappelle que toute vision sexiste et hiérarchisée de la créativité (création vs re-création) n'est pas simplement problématique d'un point de vue symbolique, mais qu'elle sous-tend une lutte pour la paternité des textes qui a des répercussions matérielles en terme termes de reconnaissance académique et salariale, ou de droits d'auteur. Avec comme agenda de libérer la traductologie et les traductrices du joug des préjugés limitants de la binarité et de la hiérarchie des sexes (hommes/femmes) et des œuvres (texte original/texte dérivé) et de la création (calque/belle infidèle), Lori Chamberlain remet également en cause une vision finalement primitive des rapports anthropologiques qui seraient basés, comme l'a été la colonisation, sur la convoitise, la cupidité, la concupiscence et la violence, et qui sont véhiculés depuis longtemps dans les métaphores de la traduction construites autour de "l'échange de mots, de femmes, de biens " (Levi-Strauss). Elle va plus loin en proposant dans ce texte un véritable programme qui permet à la traductologie féministe de sortir des seuls champs de la littérature ou de la philosophie pour dialoguer avec d'autres disciplines comme l'histoire ou la sociologie.

In 1988, in "Gender and the Metaphorics of Translation », Chamberlain revisits the figure of translation through several centuries of metaphors in translation studies. Based on the study of texts and anthologies from 1958 to 1985, particularly major texts such as those by Serge Steiner and Serge Gavronsky (and by those who inspired them from 1684 like Roscommon, Franklin or Cowper), she draws on Jacques Derrida, Terry Eagleton, Joseph Graham, Carole Maier, Suzanne Jill Levine or Susan Gubar, by deepening their approach, to analyze both the subordinate status given to translation by great translation scholars in the face of writing, but also the sexualized and submissive relationship it is supposed to have with the original text. This analysis, written during the emergence of feminist translation studies across the Atlantic, challenges a masculine and sexist conception of translation and creativity written about in terms of domination, power, gender and violence. By removing the seductive mask of stylistic beauty from the texts by translation scholars such as Steiner or Gavronsky, she reminds us that any sexist and hierarchical vision of creativity (creation vs. re-creation) is not simply problematic from a symbolic point of view, but that it underlies a struggle for authorship of texts that has material repercussions in terms of academic and salary recognition, or copyrights. With the agenda of freeing translation studies, the female translator and all creative acts from the yoke of limiting prejudices such as the binarity and hierarchy of the sexes (men/women) of works (original text/ derived text) and of creation (calque/belles infidèles), Lori Chamberlain also questions an ultimately primitive, vision of anthropological relationships that are supposed to be based, as colonization was, on lust, greed, lust and violence, and that have long been conveyed in the metaphors of translation built around "the exchange of words, women and goods" (Lévi-Strauss). She goes further by proposing a real program for feminist translation studies that enable a dialogue with disciplines other than literature or philosophy: such as history or sociology. 
INDEX

Thèmes : Recherches

Keywords : creation, translation studies, feminism, gender, sexism

Mots-clés : création, féminisme, traduction, genre, sexisme

\section{AUTEURS}

\section{LORI CHAMBERLAIN}

Après une thèse intitulée Afterwords : Translation as Poetics in Postmodern Writing, soutenue à l'université de Californie à Irvine, Lori Chamberlain devint docteure de Littérature comparée en 1982. Elle ne consacra ensuite qu'un seul article à la problématique croisée du genre et de la traduction. Cet unique article, « Gender and the metaphorics of translation » fut publié dans Signs en 1988 et sa traduction en français est proposé pour la première fois ici. Il a bouleversé les translation studies aux États-Unis et la traductologie en France, en leur apportant une nouvelle grille d'analyse féministe, qui s'inspire à la fois de traductologues féministes comme Barbara Godard ou Suzanne Jill Levine, mais aussi de Jacques Derrida. Lori Chamberlain a publié des articles sur la poésie, la fiction mais aussi sur les aspects pédagogiques de l'enseignement et la reconnaissance académique. 